\title{
In vitro fertilization (IVF): a review of 3 decades of clinical innovation and technological advancement
}

\author{
Jeff Wang \\ Mark V Sauer \\ Division of Reproductive \\ Endocrinology and Infertility, \\ Department of Obstetrics \& \\ Gynecology, College of Physicians \& \\ Surgeons, Columbia University, New \\ York, NY, USA
}

\begin{abstract}
In vitro fertilization, popularly referred to as IVF, has captured the attention of the public since its sensational introduction in 1978. Today assisted reproductive technology is available throughout most of the civilized world, and the practice is largely different from that used during the early days. Refinements in laboratory technology and clinical practice have allowed IVF to evolve into a medical procedure that is efficient, safe, readily accessible, and relatively affordable. More than 2 million IVF children have been born to date, and it is likely that continued enhancements will widen its appeal and applicability.
\end{abstract}

Keywords: IVF, assisted reproduction

\section{Introduction}

The birth of Louise Brown in 1978 was the culmination of decades of scientific research in reproductive medicine. Since then, an abundance of breakthroughs in both clinical medicine and basic science have allowed increasing numbers of infertile couples the chance to have a baby (Figure 1). To date, more than 2 million babies have been born worldwide through assisted reproductive technologies (ART). The most recent Centers for Disease Control (CDC) statistics noted that 48000 babies were born in the US and over 100000 ART cycles were performed in the year 2003 alone (CDC 2005).

\section{The early days of IVF}

Prior to 1978, women without functioning fallopian tubes were largely considered to be sterile by their physicians. At least one patent fallopian tube is necessary for natural fertilization of an oocyte by sperm in vivo. In the past, many women with damaged tubes resorted to reparative surgery, or tuboplasty in hopes of re-establishing a conduit for gametes to transit. Unfortunately, often these surgeries failed.

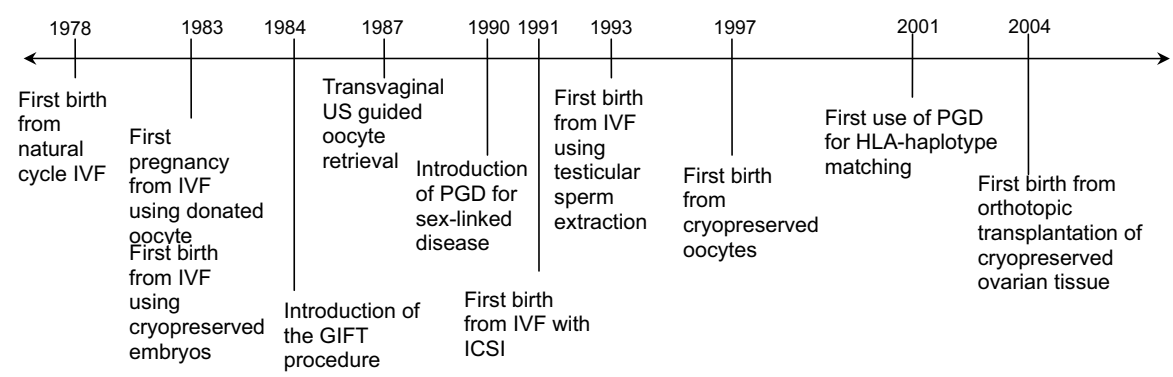

Figure I Timeline of major milestones in assisted reproductive technologies.

Abbreviations: GIFT, gamete intrafallopian transfer; HLA, human leukocyte antigen; ICSI, intracytoplasmic sperm injection; IVF, in virto fertilizations; PGD, preimplantation genetic diagnosis. 
In the late 1970's Lesley Brown, a patient with nine years of primary infertility secondary to tubal occlusion, sought the assistance of Patrick Steptoe and Robert Edwards at the Oldham General Hospital in England. At that time, fertilization of oocytes outside the human body, a process known as in vitro fertilization (IVF), was considered entirely experimental and when attempted had only resulted in miscarriages and an unsuccessful pregnancy in the fallopian tube (Steptoe and Edwards 1976). Without using medications to stimulate her ovaries, Lesley Brown underwent laparoscopic egg retrieval, with her single egg fertilized in the laboratory, and later transferred back into the uterus. The embryo transfer resulted in the first live birth from IVF, a daughter Louise Brown, who was born in July 1978 (Steptoe and Edwards 1978).

Following this sentinel and critically important event, Steptoe and Edwards, as well as several other contemporary scientists, not only successfully repeated this clinical achievement but went on to further improve and refine their pioneering efforts. The initial experience with unstimulated cycles by Edwards, Steptoe, and Purdy (Edwards et al 1980) yielded on average 0.7 oocytes per retrieval and an overall pregnancy rate of $6 \%$ per initiated cycle $(4 / 65)$. Stimulated IVF cycles with human menopausal gonadotropin (hMG) prior to laparoscopic egg retrieval was extensively studied at the Jones Intitute (Jones et al 1982; Garcia et al 1983a, 1983b). Its widespread use led to dramatic improvement in oocyte yield per retrieval and pregnancy rates. Between 1980 and 1983, the use of hMG with IVF resulted in an average recovery of 2.1-2.6 oocytes per retrieval and increasing pregnancy rates of $23.5 \%$ per retrieval in 1982 and $30 \%$ in 1983 (Edwards and Steptoe 1983).

Premature ovulation due to multi-follicular development became a prevalent problem with the increasing use of $\mathrm{hMG}$ for ovulation induction. Approximately $20 \%$ of IVF cycles were cancelled due to premature surge of luteinizing hormone (Elter and Nelson 2001). Pituitary desensitization by administration of gonadotropin releasing hormone agonist (GnRHa) prior to ovarian stimulation with hMG was first reported in 1984 (Porter et al 1984). Effective suppression of the pituitary gonadotrophes with this protocol decreased the incidence of premature ovulation to about $2 \%$ and significantly improved overall pregnancy rates with IVF (Elter and Nelson 2001). However, pituitary suppression with GnRHa also contributed to the rising incidence of potentially life threatening ovarian hyperstimulation syndrome (OHSS) in susceptible individuals by permitting more aggressive ovarian stimulation protocols without the limitation of premature ovulation (Golan et al 1988; Rizk and Smitz 1992; Nugent et al 2000).

\section{Oocyte donation}

While advances in early IVF refined the technology for treating women with tubal disease, those with natural or premature ovarian failure had no effective fertility treatments until 1983. In December of that year, a 25 year old patient with secondary amenorrhea and premature ovarian failure became the first person to successfully deliver a pregnancy using a donor egg. Dr. Peter Renou of the Monash IVF group in Australia inseminated a single oocyte, donated by a 29year-old patient undergoing IVF herself for tubal disease, with the sperm from the recipient's husband. The embryo was transferred back into the uterus of the recipient and resulted in a healthy full term live-birth (Lutjen et al 1984).

Over the last two decades, the predominant indication for oocyte donation has shifted from women with premature ovarian failure to mostly women of advanced reproductive age. Factors responsible for this trend relate to the changing demographic of the population at large. More women are delaying childbearing to pursue education and careers, marriages are occurring later in life, divorce and remarriage are more common, and effective contraception and available abortion services have eliminated many unintended pregnancies. For the older patient, traditional IVF remains an option, however pregnancy rates decline precipitously after 36 years of age, mostly due to the age associated decline in normal oocytes (Figure 2). In contrast, pregnancy rates in women using donor oocytes are known to be as high as $50 \%$ per embryo transfer in recipients across all age groups (CDC 2005). Indeed, women in their sixties have also given birth with donor oocytes, demonstrating that the postmenopausal uterus maintains the capacity to support pregnancies if provided adequate hormonal support (Antinori et al 1995; Paulson et al 1997). However, oocyte recipients experience increased obstetrical complications such as pregnancy induced hypertension (16\%-40\%), cesarean section $(40 \%-76 \%)$, and gestational diabetes (20\%) (Soderstrom Anttila 2001; Sheffer-Mimouni et al 2002; Paulson et al 2002).

The success of IVF with donor oocytes not only crossed the traditional boundaries of ART, but also unleashed a barrage of unprecedented social, ethical, and legal concerns. Debates regarding donor anonymity, financial compensation for donor participation, the need for a registry of births from third party reproduction, and age limitation on recipients of donor gametes continue to stir controversy. Despite these 


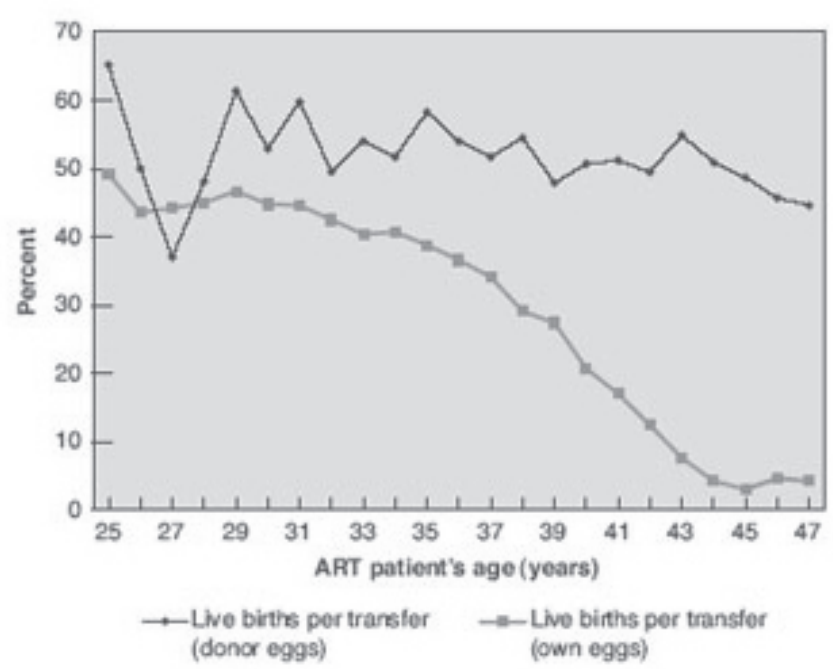

Figure 2 Live births per transfer for assisted reproductive technology (ART) cycles using fresh embryos from own and donor eggs, by ART patient's age. Copyright (C) 2005. Reproduced with permission from National Center for Chronic Disease Prevention and Health Promotion. 2005. 2003 Assisted reproductive technology success rates: National summary and fertility clinic reports.

unresolved issues, donor IVF remains an integral part of modern ART, and accounts for $11.6 \%$ of the IVF cycles performed in the US (CDC 2005).

\section{Embryo cryopreservation}

Clinical and laboratory methodology used for ART continued to evolve and improve, and a surplus of embryos in excess to what is used or needed for the initial IVF treatment became increasingly commonplace. During the early days of IVF, options for the patient with supernumerary embryos included discarding them, donating them to another infertile couple, or donating them for use in experimental research. Although cryopreservation of the embryos was an option, the freezing and thawing processes often caused permanent injury to the cells, and most embryos did not survive. This is best reflected in the very low rates of pregnancy seen following the transfer of frozen/thawed embryos throughout the 1980s. Intense efforts to develop various freezing/thawing techniques and cryoprotective agents eventually resulted in the first reported human pregnancy from a frozen embryo in 1983, which unfortunately ended in premature rupture of the membranes and termination of pregnancy at 24 weeks of gestation (Trounson and Mohr 1983).

Despite the initial set back, technology in cryopreservation continued to improve throughout the 1980s, leading to an increase in embryo survival rate and pregnancy rates. During the initial years of experimentation, at best approximately $50 \%$ of embryos survived the freeze/ thaw process and resulted in a pregnancy rate of $13.4 \%$ per embryo transfer procedure, as only $4.6 \%$ of the individual thawed embryos implanted (Friedler et al 1988). By 2003, frozen embryo transfers accounted for 21981 of the 112872 IVF cycles (17.8\%) performed in the US, with an overall live birth rate of $27.0 \%$ per embryo transfer procedure.

\section{Refinements in technique: moving away from the operating room}

During the mid-1980s, efforts to simplify and improve success rates of assisted reproduction led to the development of gamete intrafallopian transfer (GIFT) where oocytes were laparoscopically retrieved and immediately transferred into fallopian tubes along with sperm (Asch et al 1984). In addition to limiting the number of procedures to one laparoscopy, other theoretical advantages of GIFT included using the natural tubal environment for fertilization, allowing a more appropriate time of entry of the embryos into the uterine cavity, and avoidance of endometrial trauma from a transcervical embryo transfer. During this era, IVF laboratory science was still being developed, and overall success rates were low, ranging from $23.5 \%$ to $30 \%$ (Inge et al 2005). Since surgery was used to harvest gametes, the immediate replacement of the prepared specimens was attractive as it lessened the need for embryo culture and the risk of poor growth over time. However, tubal transfer was not universally applicable since patients with dense pelvic adhesions or tubal occlusion could not be treated, and patients with male factor infertility could not be observed for fertilization success or treated effectively.

Subsequently, the technique of transferring laparoscopically retrieved oocytes fertilized in vitro at the pronuclear stage into the fallopian tube via a second laparoscopy was introduced. This procedure was known as zygote intrafallopian transfer (ZIFT) and it allowed the confirmation of fertilization but maintained some of the theoretical benefits of GIFT (Hamori et al 1988). However, the use of two laparoscopies, one for oocyte retrieval and the other for zygote transfer, was a major limitation of this approach.

As the use of assisted reproductive treatments expanded from tubal infertility to include ovulation disorders, male factor infertility and decreased ovarian reserve, the number of ART cycles dramatically increased. The pioneering work reported by Steptoe and Edwards had required a surgical 
approach using laparoscopy. Subsequent modifications such as GIFT and ZIFT still relied upon laparoscopy. However, the necessity for general anesthesia and its attendant risks, along with the high overhead expense of using operating rooms provided the impetus to develop more efficient nonsurgical oocyte retrieval techniques.

Improvements in ultrasonography during the 1980s catalyzed the evolution of modern outpatient oocyte retrieval. Using transabdominal ultrasound guidance, various methods of oocyte retrieval included percutaneous (Lenz and Lauritsen 1982), transvesical (Lenz et al 1981; Lenz and Lauritsen 1982), per-urethral (Parsons et al 1985), and transvaginal follicle aspiration (Dellenbach et al 1985). Further refinements in ultrasound transducers led to the use of transvaginal ultrasound guided transvaginal follicle aspiration (Figure 3). First reported in 1987, this oocyte retrieval technique quickly became the procedure of choice due to better visualization, finer control, and less patient discomfort compared with other available methods (Wikland et al 1987). Obviating the need for laparoscopy decreased the number of personnel, time and procedure expense, reduced the risks of surgery and general anesthesia, and provided greater patient acceptance. IVF cases transitioned from 1-2 hours of hospital-based operating room time to
10-15 minute procedures that can be performed in an office setting.

\section{Comparing techniques for embryo transfer}

Multiple studies in the early 1990s comparing IVF, ZIFT, and GIFT often showed conflicting results due to multiple confounding variables inherent to different patient-selection criteria and the wide variation of practice used from clinic to clinic. While some studies showed no differences (Tanbo et al 1990; Toth et al 1992), others found that GIFT and ZIFT achieved higher pregnancy rates than IVF (Devroey et al 1989; Crosignani et al 1991; Mills et al 1992). Nevertheless, potential enhancements in outcomes of GIFT and ZIFT were marginalized by the reliance on invasive surgery, especially as IVF evolved into less expensive and minimally invasive ultrasound guided aspirations. By 1995, Society for Assisted Reproductive Technology (SART) data noted that IVF, GIFT, and ZIFT consisted of $70 \%, 6 \%$, and $2 \%$ of ART cycles respectively, despite pregnancy rates of $22.3 \%, 28.7 \%$, and $30.3 \%$ for each procedure. As the pregnancy rate of IVF improved over the last decade from $22.3 \%$ in 1995 to $33 \%$ in 2003 , the popularity of GIFT and ZIFT diminished further (CDC 2005). In 2003, GIFT and

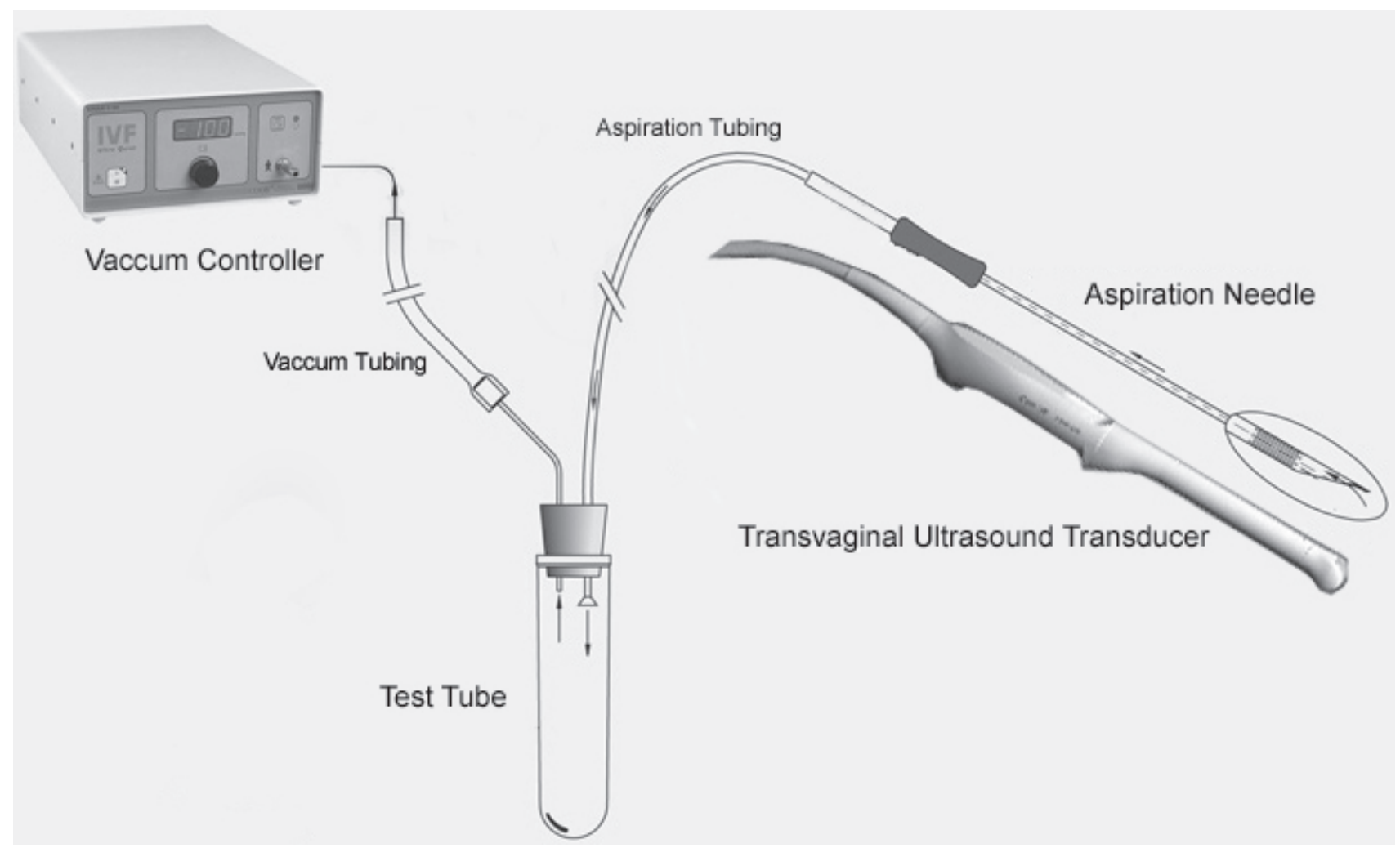

Figure 3 Schematic of modern outpatient in vitro fertilization setup. Copyright @ 2006 . Reproduced with permission from Cook Group Incorporated. 2006. Ovum aspiration diagram [online]. Accessed on 2 January 2006. URL: http://www.cookwomenshealth.com/products/infertility/I_0I/I_0I_0Ia.html. 
ZIFT were used in only $0.1 \%$ and $0.4 \%$ of ART cycles, while IVF represented the remaining $99.5 \%$ of cases (CDC 2005).

\section{Addressing the needs of infertile men}

As IVF became more commonplace in the treatment of female infertility, male infertility remained a limiting factor to overall success. Conventional IVF was much less effective when semen parameters were below the reference values for concentration (oligozoospermia), motility (asthenozoospermia), and morphology (teratozoopermia), resulting in significantly lower fertilization rates and fewer embryos available for transfer. Furthermore, azoospermic males were completely devoid of treatment options.

Several procedures were developed in the late 1980s to improve treatment fertilization problems. The first technique developed was partial zona dissection (PZD) where a small opening was made in the zona pellucida in hopes of facilitating sperm entry into the oolemma. The results of PZD were inconsistent and disappointing (Malter and Cohen 1989). Another technique introduced was subzonal insemination (SUZI) where a few motile sperm were microinjected through the perivitelline space. SUZI achieved an overall $20 \%$ fertilization rate but was still too low to be used for routine clinical application $(\mathrm{Ng}$ et al 1991; Van Steirteghem, Liu, et al 1993; Van Steirteghem, Nagy, et al 1993). Palermo and Van Steirteghem introduced a novel procedure called intracytoplasmic sperm injection (ICSI), where a single spermatozoon was microinjected into the oocyte after passage through the zona pellucida and the membranes of the oocyte (Palermo et al 1992). This procedure achieved fertilization rates of approximately $60 \%-70 \%$ when using ejaculated sperm, a rate significantly greater than SUZI or PZD and equivalent to fertilization rates experienced by men with normal parameters using conventional methods. The first pregnancies using embryos generated by ICSI were reported in 1992 (Palermo et al 1992) and the procedure has been applied increasingly from $11 \%$ of IVF cycles in 1995 to $55.6 \%$ in 2003 (CDC 2005).

ICSI not only overcame the fertilization barrier presented by oligo-, astheno-, and teratozoospermia, it also created a new possibility for azoospermic men to achieve fertility. While Temple-Smith and colleagues reported the first pregnancy by microepididymal sperm aspiration (MESA) in a patient with secondary obstructive azoospermia in 1985, epididymal sperm motility was generally low and outcomes were poor compared with that of ejaculated sperm (TempleSmith et al 1985). The advent of ICSI markedly improved fertilization rates with epididymal sperm, making the combination of MESA-ICSI widely-used procedures for patients with congenital bilateral absence of vas deferens or secondary obstructive azoospermia. Subsequently, modified percutaneous sperm aspiration (PESA) performed with needle aspiration of the epididymis through a $1 \mathrm{~cm}$ scrotal incision, was developed as an alternative to MESA which required unilateral hemiscrototomy to allow dissection, exploration, and aspiration of the epididymis with an operating microscope (Shrivastav et al 1994). Comparatively, PESA is cheaper, more acceptable to patients, has less postoperative morbidity than MESA, but retrieves fewer sperm.

When there are no motile sperm in the ejaculate or in the proximal epididymis, sperm can be extracted directly from testicular tissue by either blind needle puncture or open tissue excision. Craft et al (1993) reported successful ICSI fertilization with testicular sperm in 1993, but no pregnancy occurred. The first successful pregnancy was reported in that year (Schoysman et al 1993). Fertilization rates as high as $70 \%$ can be achieved with testicular sperm extraction (TESE) despite only using a few poor-quality sperm. Pregnancies have even been achieved in conditions such as Klinefelter syndrome, generally associated with germ cell atrophy and fibrotic, hyalinized seminiferous tubules (Staessen et al 1996). To date, there have been 39 reports of healthy children born after ICSI procedures with spermatozoa retrieved from nonmosaic Klinefelter patients since the first pregnancy in 1996 (Denschlag et al 2004).

As we enter the second decade using ICSI, debates continue on the implications of altering the process of natural selection. Inheritable causes of male fertility such as constitutional chromosomal aberrations, cystic fibrosis transmembrane conductance regulator gene mutation, or AZF deletion on the Y chromosome may be inadvertently transmitted via ICSI. In addition, potential damage to the cytoplasmic organelles of oocytes inflicted by the procedure may increase the risk of health problems in children. In a case series of 1586 ICSI established pregnancies, prenatal diagnosis showed a significantly higher percentage of de novo sex and autosomal chromosomal abnormalities (2.1\%) when sperm concentration was $<20 \times 10^{6} \mathrm{sperm} / \mathrm{ml}$ as compared with $0.24 \%$ if the concentration was $\geq 20 \times 10^{6} \mathrm{sperm} / \mathrm{ml}$ (Bonduelle et al 2002). In addition, Hansen et al (2002) found the prevalence of major birth defects in infants conceived after ICSI, as well as IVF, were doubled 
compared with infants conceived naturally. Furthermore, a multi-center study with a five year follow-up period also showed a significantly increased risk of malformation in children born after ICSI compared with that of naturally conceived children, particularly in the genitourinary system of male children (Bonduelle et al 2005). Nevertheless, a meta-analysis comparing birth defects among ICSI-births and standard IVF-births failed to attribute additional risks to the ICSI procedure, above the baseline risk associated with IVF itself (Lie et al 2005). Hence, the current available evidence suggests the increased risk of congenital malformation associated with IVF is not attributable to ICSI, per se, and may be related to other factors such as patient characteristics, ovarian stimulation protocols, or embryo culture environment.

Assessment of the developmental outcome of children at 24-28 months of age by Bayley Scale shows no significant differences between the ICSI and IVF group (Bonduelle et al 2003) In addition, motor and cognitive development are equivalent among IVF-conceived, ICSI conceived, and naturally conceived children at the age of five (Ponjaert-Kristoffersen et al 2005). Nevertheless, there is evidence for increased incidence of otherwise rare and sporadic imprinting disorders such as BeckwithWiedeman and Angelman syndrome in ICSI children, possibly due to disruption of methylation in the maternal genome or early embryo by the procedure (Sutcliffe et al 1995; Staessen et al 1996; Cox et al 2002). Another study also found an increase in the incidence of BeckwithWiedman syndrome among children born with ART but independent of ICSI (Maher et al 2003). This implies that the embryo culture condition alone may alter DNA methylation and imprinting, as has been shown by in vitro mouse embryo studies (Khosla et al 2001). Interestingly, Ludwig et al (2005) found a similar increase in the incidence of Angelman syndrome between children born to subfertile couples whose time to natural conception was greater than two years and children born to subfertile couples who underwent ART, regardless of ICSI. These findings suggest that imprinting defects and subfertility may have a common cause, and superovulation with ART in this population may further increase the risk of conceiving children with imprinting defect. Due to the overall rarity of these syndromes, large-scale systematic studies are needed to clarify the link between genomic imprinting defects and ART as well as to establish the exact biological basis of any such link.

\section{IVF and preimplantation genetic diagnosis}

Prior to 1990, options to prevent transmission of genetic defects were limited to performing chorionic villus sampling or amniocentesis and offering abortion if the fetus were found to be affected. The 3 to 5 day window between oocyte fertilization and embryo transfer provided a new opportunity to delineate which embryos are unaffected by a specific single gene disorder or chromosomal imbalance prior to transfer into the uterus. The first clinical application of this procedure called preimplantation genetic diagnosis (PGD) was used in 1990 to prevent the transmission of two Xlinked conditions: adrenoleukodystrophy and X-linked mental retardation (Handyside et al 1990). These embryos were biopsied on day 3 in vitro at the six to ten cell stages by first drilling a hole into the zona pellucida with acid Tyrodes and then aspirating blastomeres with micropipettes for analysis. Male DNA was identified by amplifying a short fragment of a Y-specific repeat sequence utilizing polymerase chain reaction (PCR). Cells not containing the amplification were presumed as female, and the embryos of origin were transferred back into the uterus to prevent transmission of the $\mathrm{X}$-linked diseases. The first application of PGD resulted in two sets of healthy female twins (Handyside et al 1990).

Following this, PCR-based tests amplifying DNA fragments with causative mutations specific for single gene disorders such as $\alpha-1$ antitrypsin deficiency and cystic fibrosis allowed identification of unaffected embryos and resulted in normal children (Verlinsky et al 1990; Handyside et al 1992). Biopsies of polar bodies during meiosis rather than blastomeres during the cleavage stage were used in some of these cases. Although this approach may be less disruptive to the embryo, it only allowed analyses of maternal DNA and hence had limited clinical applications. In the last 15 years, the number of inherited disorders diagnosed at the preimplantation stage has expanded to over 40 diseases, and the advent of multiplex-PCR which simultaneously amplifies several DNA fragments in a single reaction greatly improved the accuracy of the analyses (Kuliev et al 1998).

In additional to single gene mutations, chromosomal screening represents another application of PGD, in an attempt to decrease the risk of transferring aneuploid embryos. Initial techniques to identify $\mathrm{X}$ - and $\mathrm{Y}$ chromosomes using PCR was quickly supplanted by fluorescence in situ hybridization (FISH) due to its speed 
and capacity to identify multiple chromosomes simultaneously (Delhanty et al 1993; Munne et al 1993; Griffin et al 1994).

Today, FISH remains the primary method for routine aneuploidy screening but has expanded up to 9 chromosomes per embryo- X, Y, 13, 15, 16, 17, 18, 21, and 22. Aneuploidy screening (AS) is currently offered to various patient groups including women of advanced reproductive age, carriers of chromosomal rearrangements, and patients with history of recurrent pregnancy losses or unexplained repeated IVF failures (Munne and Wells 2002).

Evidence supporting the benefit of aneuploidy screening by PGD in the abovementioned patient groups remains equivocal. While some studies indicate that PGD-AS increases the embryo implantation rate and reduces the miscarriage rate (Gianaroli et al 2002; Munne et al 2003), a prospective randomized controlled trial comparing the outcome after blastocyst transfer combined with aneuploidy screening with PGD using fluorescence in situ hybridization (FISH) for the chromosomes X, Y, 13, 16, 18, 21, and 22 in women with advanced reproductive age ( $\geq 37$ years old) with a control group without PGD-AS showed no improvement in implantation or pregnancy rate (Staessen et al 2004). A recent meta-analysis also failed to show significant increases in pregnancy rates using PGD for aneuploidy screening (Twisk et al 2006). More prospective randomized studies are needed to determine the efficiency of PGD-AS.

\section{Expanding applications beyond infertility HLA-typing for stem cell donation}

Recent indications for PGD have expanded to genetic disorders that have a late onset such as Alzheimer's disease (Verlinsky et al 2002), Huntington disease (Sermon et al 1998), and cancer predisposition (Ao et al 1998). Another new application is human leukocyte antigen (HLA) matching first used to cure a child with Fanconi's anemia by transplanting stem cells derived from the umbilical cord blood of an unaffected sibling originating from an embryo analyzed by PGD and known to have matching HLA typing but without Fanconi's anemia mutation (Verlinsky et al 2001). Since then, similar approaches to obtain stem cell from siblings for transplantation in couples with an affected offspring have been used for acute lymphoblastic leukemia, thalasemia, and Wiscott-Aldrich (Kahraman et al 2004). These cases precipitated intense media interest and will continue to fuel ethical debates concerning "designer babies" produced for the purpose of benefiting the health and wellbeing of another.

\section{Fertility preservation for cancer patients}

Contemporary investigative efforts continue to focus on providing even more couples the opportunity to have healthy children. One such group is women diagnosed with cancer who often sustain partial or total loss of their fertility following cancer therapy. While embryo cryopreservation prior to initiating potentially gonadotoxic treatment is the most reliable method to preserve fertility, absence of a male partner or desire not to use donor sperm often precludes this approach. In such cases, oocyte cryopreservation may provide an alternative. To date, there have been approximately 100 pregnancies and 50 live-births from cryopreserved oocytes since it was first reported twenty years ago (Van der Elst 2003). The large size, high water content, and chromosomal arrangement along the meiotic spindle render metaphase-II oocytes extremely vulnerable to the intracellular ice formation during the freezing or thawing process. In addition, hardening of zona pellucida can interfere with normal fertilization process. Currently, each thawed oocyte has a mean survival rate of $47 \%$, fertilization rate of $52 \%$, but pregnancy rate of only $1.52 \%$ (Sonmezer et al 2004) based on 21 publications. Nevertheless, recent experimentation with different rates of freezing/thawing, modification of cryoprotectant from dimethylsulphoxide to 1,2-propanediol, and the addition of fertilization using ICSI has culminated in greater efficiency as reported by Fosas et al (2003) who achieved a 90\% survival rate, $75 \%$ fertilization rate, and $50 \%$ pregnancy rate.

In terms of outcome, the incidence of chromosomal abnormalities in human embryos obtained from cryopreserved oocytes is similar to that of control embryos using FISH (Cobo et al 2001). In addition, limited data based on the outcomes of 17 children resulting from cryopreserved oocytes showed no increased incidence of abnormal karyotype, preterm delivery, low birth weight, birth defects, intellectual or developmental deficits by the age of three (Winslow et al 2001).

Other methods to circumvent oocyte damage caused by the freeze/thaw process include vitrification and cryopreservation of germinal vesicles. Vitrification uses high concentration of cryoprotectants to solidify the cell into a glass-like state without the formation of ice. Based on a small study, post-thaw survival rates and pregnancy rate of 
this approach were $68.8 \%$ and $21.4 \%$, respectively, and resulted in six livebirths (Yoon et al 2003). The other alternative of freezing oocytes at the germinal vesicle stage allows higher survival rates than those frozen at the metaphase II stage (Boiso et al 2002). However, germinal vesicles need in vitro maturation (IVM) to become mature oocytes, and the inefficiency of current IVM protocol nullifies the improvement in survival rate, leading to equivalent final yield of mature oocytes as compared with that of freezing metaphase II oocytes. At this time, both nascent protocols are continuing to evolve and lack long term outcome data.

Despite the fact cryopreservation of oocytes obviates the immediate need for sperm, the inability to delay initiation of chemotherapy or the presence of estrogen sensitive malignancies precludes the use of ovarian stimulation and hence oocyte cryopreservation in many clinical scenarios. Ovarian tissue cryopreservation, achieved by biopsying and cryopreserving ovarian cortex containing primordial follicles, followed by thawing and transplanting the autograft after completion of cancer treatment offers a potential solution in those circumstances. Transplantation can be either orthotopic (in close proximity to the infundibulo-pelvic ligament) or heterotopic (ie, forearm or abdomen). To date, transient restoration of endocrine function has been reported with both approaches. Embryo transfer resulting from stimulation of cryopreserved ovarian heterotopically grafted beneath the abdominal skin did not result in pregnancy. Donnez et al (2004) reported restoration of endocrine function and a live birth following orthotopic transplantation of cryopreserved ovarian tissue in a woman who became amenorrheic after being treated for Hodgkin's lymphoma. However, some critics challenge the validity of the pregnancy arising from the cryopreserved ovarian tissue since the patient had not undergone oophorectomy. Nonetheless, these encouraging findings offer new hope to women diagnosed with cancer who desire fertility and ovarian preservation options.

\section{Conclusion}

In the three decades following the birth of Louise Brown, innovations in ART have overcome numerous seemingly insurmountable barriers to allow couples the chance to have families. Significant developments in the first decade led to greater efficiency and expanded accessibility of in vitro fertilization to the general public. In the ensuing decade, innovation and refinements in technology led to the introduction of ICSI, MESA, and TESE which provided effective treatment for male infertility. More recently, the advent of PGD provided couples with sex-linked diseases and numerous genetic disorders the ability to have children free of the condition. Efforts continue to focus on potential ways to increase the success of ART using PGD for aneuploidy screening. Finally, improving the efficiency of oocyte cryopreservation and of ovarian tissue transplantation promises to provide options to women who must delay childbearing.

Despite these major technological advances achieved by ART in the last three decades, intense efforts must be devoted to follow the long term impact of its technologies as the oldest child conceived with IVF is only twenty-sevenyears-old. Furthermore, many technologies such as ICSI, in vitro maturation, oocyte cryopreservation and vitrification, and PGD have only a smattering of data on developmental outcomes. Heightened awareness of potential health risks secondary to ovulation induction medications, in vitro culture conditions, and oocyte/embryo manipulations is paramount to the continuous surveillance of rare complications of ART that may only manifest over time. Periodic meta-analysis to pool well-conducted studies limited by sample size and statistical power may help uncover true associations between ART and infrequent diseases. Diligent maintenance of national ART birth registries and expanded access to international data will further enhance the investigations on IVF-associated birth defects and imprinting disorders.

In summary, few fields of medicine have enjoyed the popular growth and sustained improvements witnessed by physicians and their patients with infertility. However, there is increasing evidence that ART-conceived children may be at greater risk of perinatal complications than naturally conceived children and that knowledge on long-term health effects of ART is incomplete. Hence, all clinicians and researchers involved in the care of these patients must maintain a heightened awareness of these potential issues. As ART approaches its third decade, new and existing technologies must be used responsibly to help infertile couples achieve their goals without compromising the principle of 'first, do no harm'.

\section{References}

Antinori S, Versaci C, Panci C, et al. 1995. Fetal and maternal morbidity and mortality in menopausal women aged 45-63 years. Hum Reprod, 10:464-9.

Ao A, Wells D, Handyside AH, et al. 1998. Preimplantation genetic diagnosis of inherited cancer: familial adenomatous polyposis coli. $J$ Assist Reprod Genet, 15:140-4. 
Asch RH, Ellsworth LR, Balmaceda JP, et al. 1984. Pregnancy after translaparoscopic gamete intrafallopian transfer. Lancet, 2:1034-5.

Boiso I, Marti M, Santalo J, et al. 2002. A confocal microscopy analysis of the spindle and chromosome configurations of human oocytes cryopreserved at the germinal vesicle and metaphase II stage. Hum Reprod, 17:1885-91.

Bonduelle M, Ponjaert I, Steirteghem AV, et al. 2003. Developmental outcome at 2 years of age for children born after ICSI compared with children born after IVF. Hum Reprod, 18:342-50.

Bonduelle M, Van Assche E, Joris H, et al. 2002. Prenatal testing in ICSI pregnancies: incidence of chromosomal anomalies in 1586 karyotypes and relation to sperm parameters. Hum Reprod, 17:2600-14.

Bonduelle M, Wennerholm UB, Loft A, et al. 2005. A multi-centre cohort study of the physical health of 5-year-old children conceived after intracytoplasmic sperm injection, in vitro fertilization and natural conception. Hum Reprod, 20:413-19.

[CDC] National Center for Chronic Disease Prevention and Health Promotion. 2005. 2003 Assisted reproductive technology success rates: National summary and fertility clinic reports [online]. Accessed on 5 December 2005. URL: http://www.cdc.gov/ART/ART2003/PDF/ ART2003.pdf.

Cobo A, Rubio C, Gerli S, et al. 2001. Use of fluorescence in situ hybridization to assess the chromosomal status of embryos obtained from cryopreserved oocytes. Fertil Steril, 75:354-60.

Cook Group Incorporated. 2006. Ovum aspiration diagram [online]. Accessed on 2 January 2006. URL: http:// www.cookwomenshealth.com/products/infertility/1_01/ 1_01_01a.html.

Cox GF, Burger J, Lip V, et al. 2002. Intracytoplasmic sperm injection may increase the risk of imprinting defects. Am J Hum Genet, 71:162-4.

Craft I, Bennett V, Nicholson N. 1993. Fertilising ability of testicular spermatozoa. Lancet, 342:864.

Crosignani PG, Walters DE, Soliani A. 1991. The ESHRE multicentre trial on the treatment of unexplained infertility: a preliminary report. European Society of Human Reproduction and Embryology. Hum Reprod, 6:953-8.

Delhanty JD, Griffin DK, Handyside AH, et al. 1993. Detection of aneuploidy and chromosomal mosaicism in human embryos during preimplantation sex determination by fluorescent in situ hybridisation, (FISH). Hum Mol Genet, 2:1183-5.

Dellenbach P, Nisand I, Moreau L, et al. 1985. Transvaginal sonographically controlled follicle puncture for oocyte retrieval. Fertil Steril, 44:656-62.

Denschlag D, Tempfer C, Kunze M, et al. 2004. Assisted reproductive techniques in patients with Klinefelter syndrome: a critical review. Fertil Steril, 82:775-9.

Devroey P, Staessen C, Camus M, et al. 1989. Zygote intrafallopian transfer as a successful treatment for unexplained infertility. Fertil Steril, 52:246-9.

Donnez J, Dolmans MM, Demylle D, et al. Livebirth after orthotopic transplantation of cryopreserved ovarian tissue. Lancet, 364:140510.

Edwards RG, Steptoe PC. 1983. Current status of in-vitro fertilisation and implantation of human embryos. Lancet, 2:1265-9.

Edwards RG, Steptoe PC, Purdy JM. 1980. Establishing full-term human pregnancies using cleaving embryos grown in vitro. $\mathrm{Br} J$ Obstet Gynaecol, 87:737-56.

Elter K, Nelson LR. 2001. Use of third generation gonadotropin-releasing hormone antagonists in in vitro fertilization-embryo transfer: a review. Obstet Gynecol Surv, 56:576-88.

Fosas N, Marina F, Torres PJ, et al. 2003. The births of five Spanish babies from cryopreserved donated oocytes. Hum Reprod, 18:1417-21.

Friedler S, Giudice LC, Lamb EJ. 1988. Cryopreservation of embryos and ova. Fertil Steril, 49:743-64.

Garcia JE, Jones GS, Acosta AA, et al. 1983a. Human menopausal gonadotropin/human chorionic gonadotropin follicular maturation for oocyte aspiration: phase I, 1981. Fertil Steril, 39:167-73.
Garcia JE, Jones GS, Acosta AA, et al. 1983b. Human menopausal gonadotropin/human chorionic gonadotropin follicular maturation for oocyte aspiration: phase II, 1981. Fertil Steril, 39:174-9.

Gianaroli L, Magli MC, Ferraretti AP, et al. 2002. The role of preimplantation diagnosis for aneuploidies. Reprod Biomed Online, 4(Suppl 3):31-6.

Golan A, Ron-El R, Herman A, et al. 1988. Ovarian hyperstimulation syndrome following D-Trp-6 luteinizing hormone-releasing hormone microcapsules and menotropin for in vitro fertilization. Fertil Steril, 50:912-16.

Griffin DK, Handyside AH, Harper JC, et al. 1994. Clinical experience with preimplantation diagnosis of sex by dual fluorescent in situ hybridization. J Assist Reprod Genet, 11:132-43.

Hamori M, Stuckensen JA, Rumpf D, et al. 1988. Zygote intrafallopian transfer (ZIFT): evaluation of 42 cases. Fertil Steril, 50:519-21.

Handyside AH, Kontogianni EH, Hardy K, et al. 1990. Pregnancies from biopsied human preimplantation embryos sexed by Y-specific DNA amplification. Nature, 344:768-70.

Handyside AH, Lesko JG, Tarin JJ, et al. 1992. Birth of a normal girl after in vitro fertilization and preimplantation diagnostic testing for cystic fibrosis. $N$ Engl J Med, 327:905-9.

Hansen M, Kurinczuk JJ, Bower C, et al. 2002. The risk of major birth defects after intracytoplasmic sperm injection and in vitro fertilization. N Engl J Med, 346:725-30.

Inge GB, Brinsden PR, Elder KT. 2005. Oocyte number per live birth in IVF: were Steptoe and Edwards less wasteful? Hum Reprod, 20:588-92.

Jones HW Jr, Jones GS, Andrews MC, et al. 1982. The program for in vitro fertilization at Norfolk. Fertil Steril, 38:14-21.

Kahraman S, Karlikaya G, Sertyel S, et al. 2004. Clinical aspects of preimplantation genetic diagnosis for single gene disorders combined with HLA typing. Reprod Biomed Online, 9:529-32.

Khosla S, Dean W, Brown D, et al. 2001. Culture of preimplantation mouse embryos affects fetal development and the expression of imprinted genes. Biol Reprod, 64:918-26.

Kuliev A, Rechitsky S, Verlinsky O, et al. 1998. Preimplantation diagnosis of thalassemias. J Assist Reprod Genet, 15:219-25.

Lenz S, Lauritsen JG. 1982. Ultrasonically guided percutaneous aspiration of human follicles under local anesthesia: a new method of collecting oocytes for in vitro fertilization. Fertil Steril, 38:673-7.

Lenz S, Lauritsen JG, Kjellow M. 1981. Collection of human oocytes for in vitro fertilisation by ultrasonically guided follicular puncture. Lancet, 1:1163-4.

Lie RT, Lyngstadass A, Orstavik KH, et al. 2005. Birth defects in children conceived by ICSI compared with children conceived by other IVFmethods; a meta-analysis. Int J Epidemiol, 34:696-701.

Ludwig M, Katalinic A, Gross S, et al. 2005. Increased prevalence of imprinting defects in patients with Angelman syndrome born to subfertile couples. J Med Genet, 42:289-91.

Lutjen P, Trounson A, Leeton J, et al. 1984. The establishment and maintenance of pregnancy using in vitro fertilization and embryo donation in a patient with primary ovarian failure. Nature, 307:174-5.

Maher ER, Brueton LA, Bowdin SC, et al. 2003. Beckwith-Wiedemann syndrome and assisted reproduction technology (ART). J Med Genet, 40:62-4.

Malter HE, Cohen J. 1989. Partial zona dissection of the human oocyte: a nontraumatic method using micromanipulation to assist zona pellucida penetration. Fertil Steril, 51:139-48.

Mills MS, Eddowes HA, Cahill DJ, et al. 1992. A prospective controlled study of in-vitro fertilization, gamete intra-fallopian transfer and intrauterine insemination combined with superovulation. Hum Reprod, 7:490-4.

Munne S, Sandalinas M, Escudero T, et al. 2003. Improved implantation after preimplantation genetic diagnosis of aneuploidy. Reprod Biomed Online, 7:91-7.

Munne S, Weier HU, Stein J, et al. 1993. A fast and efficient method for simultaneous $\mathrm{X}$ and $\mathrm{Y}$ in situ hybridization of human blastomeres. $J$ Assist Reprod Genet, 10:82-90. 
Munne S \& Wells D. 2002. Preimplantation genetic diagnosis. Curr Opin Obstet Gynecol, 14:239-44.

Ng SC, Bongso A, Ratnam SS. 1991. Microinjection of human oocytes: a technique for severe oligoasthenoteratozoospermia. Fertil Steril, $56: 1117-23$

Nugent D, Vandekerckhove P, Hughes E, et al. 2000. Gonadotrophin therapy for ovulation induction in subfertility associated with polycystic ovary syndrome. Cochrane Database Syst Rev, 4:CD000410.

Palermo G, Joris H, Devroey P, et al. 1992. Pregnancies after intracytoplasmic injection of single spermatozoon into an oocyte. Lancet, 340:17-18.

Parsons J, Riddle A, Booker M, et al. 1985. Oocyte retrieval for in-vitro fertilisation by ultrasonically guided needle aspiration via the urethra. Lancet, 1:1076-7.

Paulson RJ, Boostanfar R, Saadat P, et al. 2002. Pregnancy in the sixth decade of life: obstetric outcomes in women of advanced reproductive age. JAMA, 288:2320-23

Paulson RJ, Thornton MH, Francis MM, et al. 1997. Successful pregnancy in a 63-year-old woman. Fertil Steril, 67:949-51.

Ponjaert-Kristoffersen I, Bonduelle M, Barnes J, et al. 2005. International collaborative study of intracytoplasmic sperm injection-conceived, in vitro fertilization-conceived, and naturally conceived 5-year-old child outcomes: cognitive and motor assessments. Pediatrics, 115:e283-9.

Porter RN, Smith W, Craft IL, et al. 1984. Induction of ovulation for in-vitro fertilisation using buserelin and gonadotropins. Lancet, 2:1284-5.

Rizk B, Smitz J. 1992. Ovarian hyperstimulation syndrome after superovulation using GnRH agonists for IVF and related procedures. Hum Reprod, 7:320-7.

Schoysman R, Vanderzwalmen P, Nijs M, et al. 1993. Pregnancy after fertilisation with human testicular spermatozoa. Lancet, 342:1237.

Sermon K, Goossens V, Seneca S, et al. 1998. Preimplantation diagnosis for Huntington's disease (HD): clinical application and analysis of the HD expansion in affected embryos. Prenat Diagn, 18:1427-36.

Sheffer-Mimouni G, Mashiach S, Dor J, et al. 2002. Factors influencing the obstetric and perinatal outcome after oocyte donation. Hum Reprod, 17:2636-40.

Shrivastav P, Nadkarni P, Wensvoort S, et al. 1994. Percutaneous epididymal sperm aspiration for obstructive azoospermia. Hum Reprod, 9:2058-61.

Soderstrom Anttila V. 2001. Pregnancy and child outcome after oocyte donation. Hum Reprod Update, 7:28-32.

Sonmezer M, Oktay K. 2004. Fertility preservation in female patients. Hum Reprod Update, 10:251-66.

Staessen C, Coonen E, Van Assche E, et al. 1996. Preimplantation diagnosis for $\mathrm{X}$ and $\mathrm{Y}$ normality in embryos from three Klinefelter patients. Hum Reprod, 11:1650-3.

Staessen C, Platteau P, Van Assche E, et al. 2004. Comparison of blastocyst transfer with or without preimplantation genetic diagnosis for aneuploidy screening in couples with advanced maternal age: a prospective randomized controlled trial. Hum Reprod, 19:2849-58.
Steptoe PC, Edwards RG. 1976. Reimplantation of a human embryo with subsequent tubal pregnancy. Lancet, 1:880-2.

Steptoe PC, Edwards RG. 1978. Birth after the reimplantation of a human embryo. Lancet, 2:366.

Sutcliffe AG, D'Souza SW, Cadman J, et al. 1995. Outcome in children from cryopreserved embryos. Arch Dis Child, 72:290-3.

Tanbo T, Dale PO, Abyholm T. 1990. Assisted fertilization in infertile women with patent fallopian tubes. A comparison of in-vitro fertilization, gamete intra-fallopian transfer and tubal embryo stage transfer. Hum Reprod, 5:266-70.

Temple-Smith PD, Southwick GJ, Yates CA, et al. 1985. Human pregnancy by in vitro fertilization (IVF) using sperm aspirated from the epididymis. $J$ In Vitro Fert Embryo Transf, 2:119-22.

Toth TL, Oehninger S, Toner JP, et al. 1992. Embryo transfer to the uterus or the fallopian tube after in vitro fertilization yields similar results. Fertil Steril, 57:1110-13.

Trounson A, Mohr L. 1983. Human pregnancy following cryopreservation, thawing and transfer of an eight-cell embryo. Nature, 305:707-9.

Twisk M, Mastenbroek S, van Wely M, et al. 2006. Preimplantation genetic screening for abnormal number of chromosomes (aneuploidies) in in vitro fertilisation or intracytoplasmic sperm injection. Cochrane Database Syst Rev, 1:CD005291.

Van der Elst J. 2003. Oocyte freezing: here to stay? Hum Reprod Update, 9:463-70.

Van Steirteghem AC, Liu J, Joris H, et al. 1993. Higher success rate by intracytoplasmic sperm injection than by subzonal insemination. Report of a second series of 300 consecutive treatment cycles. Hum Reprod, 8:1055-60.

Van Steirteghem AC, Nagy Z, Joris H, et al. 1993. High fertilization and implantation rates after intracytoplasmic sperm injection. Hum Reprod, 8:1061-6.

Verlinsky Y, Ginsberg N, Lifchez A, et al. 1990. Analysis of the first polar body: preconception genetic diagnosis. Hum Reprod, 5:8269.

Verlinsky Y, Rechitsky S, Schoolcraft W, et al. 2001. Preimplantation diagnosis for Fanconi anemia combined with HLA matching. JAMA, 285:3130-3.

Verlinsky Y, Rechitsky S, Verlinsky O, et al. 2002. Preimplantation diagnosis for early-onset Alzheimer disease caused by V717L mutation. JAMA, 287:1018-21.

Wikland M, Enk L, Hammarberg K, et al. 1987. Use of a vaginal transducer for oocyte retrieval in an IVF/ET program. $J$ Clin Ultrasound, 15:245-51.

Winslow KL, Yang D, Blohm PL, et al. 2001. Oocyte cryopreservation/ a three year follow up of sixteen births [abstract]. Fertil Steril, 76(Suppl 1):S120-1.

Yoon TK, Kim TJ, Park SE, et al. 2003. Live births after vitrification of oocytes in a stimulated in vitro fertilization-embryo transfer program. Fertil Steril, 79:1323-6. 